\title{
Das alte Ägypten und die Illuminaten
}

Das 18. Jahrhundert war nicht nur das Zeitalter der Aufklärung, sondern auch der geheimen Gesellschaften - zwei gegenläufige Tendenzen, wie man annehmen sollte, würden wir doch normalerweise Aufklärung mit Öffentlichkeit und Geheimhaltung mit Obskurantismus verbinden. Einen gemeinsamen Nenner zwischen Aufklärung und Geheimnis bietet jedoch die neue politische Theorie der antiken, speziell ägyptischen Mysterienkulte, die zuerst der britische Bischof William Warburton um 1740 vorgetragen hatte, die aber ihren großen europäischen Einfluss erst eine Generation später auszuüben begann. Der englische Theologe, Altphilologe und Shakespeare-Forscher William Warburton publizierte sein monumentales Werk Die göttliche Sendung Moses in drei Bänden von 1738 bis 1741. Der zweite von neun Teilen war den Mysterien gewidmet. In Deutschland wurde das Werk in der Übersetzung von Johann Christian Schmidt, Frankfurt und Leipzig 1751-1753, bekannt. ${ }^{2}$ Den eigentlichen Durchbruch verschaffte der Theorie Warburtons aber erst der Philosoph und Historiker Christoph Meiners, der sie seinem Buch Über die Mysterien der Alten, besonders die Eleusinischen Geheimnisse zugrunde legte. ${ }^{3}$ Dieses Buch machte in den Kreisen der Freimaurer Furore und

1 William Warburton: The divine legation of Moses demonstrated on the principles of a religious deist, from the omission of the doctrine of a future state of reward and punishment in the Jewish dispensation, London 1738-1741, London, 2. Aufl. 1778.

2 Dr. William Warburton's „Göttliche Sendung Mosis“. Aus den Grundsätzen der Deisten bewiesen, übersetzt von Johann Christian Schmidt, 3 Teile, Frankfurt und Leipzig 17511753; französische Teilübersetzung von Léonard des Malpeines: Essai sur les hiéroglyphes des Égyptiens, où l'on voit l'origine et le progrès du langage et de l'écriture, l'antiquité des sciences en Égypte et l'origine du culte des animaux. Traduit de l'anglais de M. Warburton. Avec des observations sur l'antiquité des hiéroglyphes scientifiques et des remarques sur la chronologie et sur la première écriture des Chinois, 2 Bde., Paris 1744. Neuausgabe von Patrick Tort: William Warburton, Essai sur les hiéroglyphes des Égyptiens [...]. Collection Palimpseste, Paris 1978; davon eine deutsche Teilausgabe in der Übersetzung von Johann Christian Schmidt durch Peter Krumme: Versuch über die Hieroglyphen der Ägypter, Frankfurt a. M., Berlin und Wien 1980.

3 Christoph Meiners: Über die Mysterien der Alten, besonders die Eleusinischen Geheimnisse, Göttingen 1776 
wirkte als Auslöser einer wahren Flut von Publikationen, die sich mit den alten Mysterien beschäftigten. In den Jahren zwischen 1776 und 1800 erschienen vor allem in Deutschland und Österreich mehrere Dutzend gelehrter Abhandlungen zu diesem Thema, die meisten aus freimaurerischer Feder. ${ }^{4}$ Der Ingolstädter Professor Adam Weishaupt legte Meiners' Darstellung der eleusinischen Einweihung, die er übrigens, wie schon Warburton, für einen Ableger der ägyptischen hielt, seinem neugegründeten Orden der Illuminaten zugrunde. ${ }^{5}$ Auch im Bereich der schönen Literatur und der Künste griff die Mysterienfaszination um sich. Als eine Untergattung des Bildungsromans entwickelte sich der Geheimbundroman, der auf der Analogie zwischen Aufklärung und Einweihung basiert. ${ }^{6}$ Diese Analogie stellt die Verbindung her zwischen den scheinbaren Gegensätzen Aufklärung und Geheimnis. Im Vollzug der Einweihung wird der Initiand aufgeklärt über die wahre Natur der Welt und den wahren Zusammenhang der Dinge und von den Illusionen und Vorurteilen befreit, die ihn vorher in Finsternis gefangen hielten. Diese Analogie mag den enormen Erfolg der Bildungsromane im Anschluss an François de Fenélons Aventures de Télémaque (1699) erklären, von denen viele den Bildungsweg des Helden als Einweihung in die Mysterien darstellen und vorzugsweise als Reise in die ägyptische Unterwelt verlegen. Die prominentesten Beispiele für diese Mysterienfaszination in den schönen Künsten stellen die Oper Die Zauberflöte (1791) von Mozart und Schikaneder und Schillers Ballade Das verschleierte Bild zu Sais (1795) dar, ${ }^{7}$ und auch Goethes Wilhelm Meisters Lehrjahre (1795) gehört zur Gattung des Geheimbundromans.

Was ist nun das spezifisch Neue und Besondere dieser Mysterientheorie, dass sie eine solche Wirkung entfalten konnte? Das Geheimnis dieser Wirkung liegt in

4 Vgl. hierzu mein Buch: Religio Duplex. Ägyptische Mysterien und europäische Aufklärung, Berlin 2010, Kap. 4: Religio Duplex und die Freimaurerei, S. 122-154.

5 Adam Weishaupt, Jesuitenzögling und Professor für Kirchenrecht und praktische Philosophie in Ingolstadt, hat sich bei der Einrichtung des Ordens vornehmlich an den Jesuiten orientiert. Die höchste Stufe seiner dreigeteilten Hierarchie, die „Mysterienklasse“, aber mit ihrer Gliederung in „kleine“ und "große Mysterien“, ist eindeutig von Christoph Meiners' Darstellung der Eleusinien inspiriert.

6 Linda Simonis: Die Kunst des Geheimen. Esoterische Kommunikation und ästhetische Darstellung im 18. Jahrhundert, Heidelberg 2002, mit reicher Bibliographie zu diesem Zusammenhang. Zum Geheimbundroman des 18. Jahrhunderts siehe auch Rosemarie Nicolai-Haas: Die Anfänge des deutschen Geheimbundromans. In Peter Christian Ludz (Hrsg.): Geheime Gesellschaften. Wolfenbütteler Studien zur Aufklärung V/1, Heidelberg 1979, S. 267-292, und besonders Michael Voges: Aufklärung und Geheimnis, Tübingen 1987.

7 Vgl.dazu Jan Assmann: Das verschleierte Bild zu Sais. Schillers Ballade und ihre griechischen und ägyptischen Hintergründe. In: Lectio Teubneriana VIII, Stuttgart, Leipzig 1999. 
der politischen Deutung der Mysterien, ihres Ursprungs und ihrer Funktion. Damit gewann eine bis dahin rein gelehrte und antiquarische Frage auf einmal eine hochaktuelle politische Bedeutung. Die antiken Mysterien stellten sich in dieser Deutung in einem Licht dar, in dem sich die der Aufklärung verschworenen Geheimgesellschaften der Zeit wie in einem Spiegel wieder erkennen konnten.

Warburton argumentierte, dass die Mysterien gleichzeitig mit dem Staat entstanden. ${ }^{8}$ In ihnen wurde eine ursprüngliche und natürliche Form von Religion bewahrt, die nicht auf Offenbarung und heiligen Schriften, sondern auf dem Studium der Natur bzw. Schöpfung beruhte und die Natur als einzige Gottheit verehrte. Ägypten galt als der erste Staat der Menschheitsgeschichte und damit auch als die erste Kultur, die ihrer ursprünglichen Religion die Form einer Mysterienreligion gab, mit geheimen Riten und theologischen Lehren, die dann zum Modell aller späteren Mysterienreligionen wurde.

Wie hängen nun politische und religiöse Organisationsform zusammen, was zwang, mit anderen Worten, die Ägypter dazu, zugleich mit der Einrichtung des Staates ihre Religion zum Geheimnis zu machen? Warburtons Erklärung lief folgendermaßen: Die Urreligion der Natur konnte nicht staatstragend sein. Staat und zivile Gesellschaft brauchen persönliche Götter, die belohnen und strafen, Moral und Gesetze schützen und nationale und regionale Identitäten repräsentieren. Die Menschen würden die Gesetze nicht respektieren ohne Furcht vor göttlicher Strafe und verlören jede moralische und politische Orientierung ohne Stadtund Staatsgötter. Daher musste sich jede Gesellschaft, die nach sozialer Ordnung und politischer Macht strebte, solche Götter zulegen, und da sie nicht auf eine Offenbarung zurückgreifen konnte, wie sie nur den Israeliten durch Moses zuteil geworden war, mussten sie diese Götter erfinden, indem sie verdiente Gesetzgeber, Kulturgründer, Heroen, Häuptlinge und Könige zu Göttern machten und ihnen Funktionen zuwiesen in der Aufsicht über die Gesetze und der Symbolisierung politischer und sozialer Identität. Warburton gab sich daher große Mühe, seine heidnischen Kulturen vom Vorwurf des Priesterbetrugs freizuhalten, der schon in den religionskritischen Schriften der antiken Aufklärung, von Euripides bis Lu$\mathrm{krez}$, immer wieder erhoben worden war und im 18. Jahrhundert mit Bernard de Fontenelle und anderen populär gemacht wurde. Diese Götter sind zwar Fiktionen, aber legitime Fiktionen, weil sie unentbehrlich sind für die Aufrechterhaltung von Ordnung, Recht, Gerechtigkeit und Herrschaft. Man brauchte also die fiktiven Götter, wollte andererseits aber auch nicht auf die natürliche Religion

8 Vgl. hierzu im Einzelnen: Religio Duplex (wie Anm. 4), Kap. 3: Religio Duplex und politische Theologie, S. 88-120. 
und Theologie verzichten, die als Wahrheit geglaubt und durch Alter geheiligt war. Vor dieser Wahrheit mussten aber die fiktiven Götter geschützt werden, damit sie nicht als fiktiv entlarvt würden und so ihre staatstragende Glaubwürdigkeit verlören. Daher musste die natürliche Urreligion im Verborgenen praktiziert und tradiert werden.

Warburton gründete seine Theorie auf zwei antike Traditionen. Die eine besteht in dem stoischen, durch Marcus Terentius Varro bzw. dessen Wiedergabe bei Augustinus bekannt gewordenen Konzept der theologia tripertita mit seiner Unterscheidung zwischen politischer, poetischer und natürlicher Theologie, das sich im 17. Jahrhundert auf die Dichotomie von politischer und natürlicher Theologie zugespitzt hatte. ${ }^{9}$ Die andere geht auf die platonische Unterscheidung zurück zwischen der Masse der Vielen, die unfähig zu abstraktem Denken und reiner Erkenntnis, und daher auf Fabeln und Bilder angewiesen sind, und den Philosophen, die sich aus der Sphäre der populären Illusionen befreien und zum Licht der Erkenntnis aufsteigen können. Beide Traditionen - die ursprünglich stoische Dichotomie zwischen drei bzw. zwei Theologien und die ursprünglich platonische zwischen zwei Wissens- und Erkenntnisformen - wurden bereits im 17. Jahrhundert vor allem in England bei Gelehrten wie John Selden, John Spencer und Ralph Cudworth zum Konzept der doppelten Religion verschmolzen, mit einer volkstümlichen, offiziellen, polytheistischen Außenseite und einer geheimen, monooder pantheistischen, esoterischen Innenseite. ${ }^{10}$ Warburtons bahnbrechende Neuerung bestand dann aber darin, dem traditionellen Konzept der doppelten

9 Die Begriffe „politische“ und „natürliche“ Theologie bilden zusammen mit der "poetischen Theologie“ das Schema der theologia tripertita („dreigeteilten Theologie“), das über Varro auf griechische (stoische) Traditionen zurückgeht. Für die theologia politikē oder civilis sind die öffentlichen kultischen Institutionen, Tempel, Kirchen und ihre Priester zuständig, die theologia poiētikē oder fabularis ist Sache der Dichter, und die theologia physikē oder naturalis gehört den Philosophen. Zur theologia tripertita vgl. Godo Lieberg: Die theologia tripertita als Formprinzip antiken Denkens. In: Rheinisches Museum 125, 1982, S. $25-$ 53, W. Geerlings: Die theologia mythica des M. Terentius Varro. In Gerhard Binder und Bernd Effe (Hrsg.): Mythos, Trier 1990, S. 205-222. Zur theologia civilis vgl. im besonderen Hubert Cancik: Augustinus als constantinischer Theologe. In Jacob Taubes (Hrsg.): Der Fürst dieser Welt, München 1983, S. 136-152; Albrecht Dihle: Die Theologia tripertita bei Augustin. In Hubert Cancik et al. (Hrsg.): Geschichte - Tradition - Reflexion, Bd. 2, Tübingen 1996, S. 183-202. Zur antiken Begriffsgeschichte der politischen Theologie siehe Ernst Feil: Von der ,Politischen Theologie ' zur ,Theologie der Revolution'? In Ernst Feil und Rudolf Weth (Hrsg.): Diskussion zur Theologie der Revolution, München und Mainz 1969, S. 110-132, hier S. 113 ff.

10 Vgl. hierzu Religio Duplex, Kap. 2 (wie Anm. 4): Vom Doppelsinn der Zeichen zur Doppelten Religion, S. 63-86. 
Religion eine politische Deutung zu geben und damit beiden Seiten der heidnischen Religionen eine Art von Legitimität zuzugestehen: der exoterischen durch ihre politische Funktion, für Ordnung, Moral, Sinn und Sicherheit zu sorgen, und der esoterischen Seite durch ihren Bezug auf die Urreligion der Natur, die natürliche Offenbarung des Schöpfers.

Natürlich sind diese Theorien sowohl der doppelten Religion als auch ihrer politischen Deutung ohne jede historische Bedeutung, was das alte Ägypten angeht. Das Bild, das sich diese Zeit vom alten Ägypten als einer gespaltenen Kultur machte, geteilt in eine esoterische und eine exoterische Religion, ist wichtig, nicht weil es irgendwelche authentischen Informationen über das alte Ägypten enthielt, sondern weil es der zeitgenössischen Kultur und Gesellschaft als ein Spiegel diente, um sich über ihre eigene Lage zu verständigen. Die Tatsache, dass man den Staat mit dem Imaginären und Fiktiven, die Sphäre des Geheimnisses dagegen mit Wahrheit und Aufklärung verband, ist zwar abwegig mit Bezug auf die Antike, aber umso aufschlussreicher für die Sozial- und Geistesgeschichte des 18. Jahrhunderts. ${ }^{11}$

Im Zentrum des Interesses dieser Zeit am alten Ägypten und an den alten Mysterien stand die Initiation. Nicht nur die allgemeine Idee der doppelten Religion oder gespaltenen Kultur, sondern vor allem auch der Weg, der von der exoterischen zur esoterischen Seite führte und die Verwandlung, die der Initiand auf diesem Weg durchzumachen hatte, waren Gegenstand der Faszination. Die antiken Quellen stellten die ägyptische Initiation unter zwei Aspekten dar, einem grammatologischen und einem topologischen. Der grammatologische Aspekt betonte die Existenz zweier scheinbar grundverschiedener Schriftsysteme, der bildhaften Hieroglyphen und der abstrakten Kursivschrift, der topologische Aspekt gründete sich auf die Fülle und Ausdehnung der unterirdischen Anlagen und bezog die oberirdischen Bauten auf die öffentliche Volksreligion und die unterirdischen auf die Geheimreligion. Beide Aspekte basierten auf Missverständnissen: Die beiden Schriften unterscheiden wir heute als Varianten desselben Schriftsystems (im Sinne von Monumentalschrift und Kursivschrift), und die unterirdischen Anlagen sind nach heutigem Wissen Grabanlagen.

11 Reinhart Koselleck: Kritik und Krise. Eine Studie zur Pathogenese der bürgerlichen Welt, Freiburg 1959, Helge Jordheim: Die Hypokrisie der Aufklärer - oder: War Wieland ein Lügner? Eine Untersuchung zu Kosellecks Kritik und Krise. In Jussi Kurunmäki und Kari Palonen (Hrsg.): Time, History and Politics/ Zeit, Geschichte und Politik. Zum achtzigsten Geburtstag von Reinhart Koselleck, Jyväskylä 2003, S. 35-54. 
Auf den grammatologischen Aspekt will ich hier nur kurz eingehen. ${ }^{12}$ In Ägypten verschwand die Kenntnis der Hieroglyphenschrift im Lauf des 4. Jahrhunderts, aber eine Fülle von Informationen überdauerte in griechischen Texten. Die Griechen waren von der ägyptischen Schriftkultur aus zwei Gründen fasziniert. Der eine bestand in dem ikonischen Charakter der Hieroglyphen als einer Bilderschrift und ihrem scheinbaren Bezug, nicht auf Sprache, sondern auf Dinge und Konzepte, der andere lag in der Verwendung zweier scheinbar verschiedener Schriften, von denen man die Bilderschrift als Geheimschrift und die Kursivschrift nicht nur als Alltags-, sondern auch als Alphabetschrift deutete. Für die abendländische Ägyptenrezeption war der grammatologische Ägyptendiskurs enorm einflussreich. Die Passagen, die den ikonischen Charakter der Hieroglyphen herausstellten, betonten drei Punkte: die Nicht-Diskursivität (die Hieroglyphen geben in ihrer Anordnung nicht die Abfolge der Silben und Wörter wieder), den metaphorischen Charakter (die dargestellten Gegenstände beziehen sich auf metaphorischem Wege auf Konzepte und anderes, so dass man mit einem zwar enorm großen, aber nicht unbegrenzten Bilderinventar alles schreiben kann) und die damit verbundene Bedeutung von Weltwissen und Gedächtnis für die Schriftbeherrschung. Die Beherrschung der Schrift erforderte ein ungeheures Wissen über die metaphorische und damit geheime Bedeutung der Dinge. Dadurch kam das Lesen- und Schreibenlernen einer Initiation in die Geheimnisse der Natur gleich.

Für die Alltagszwecke schriftlicher Kommunikation erfanden die Ägypter eine zweite Schrift, die leicht zu lernen und ohne geheime Bedeutungen war. Die Unterscheidung zweier Schriften kommt am klarsten bei Diodor zum Ausdruck, der seinerseits Hekataios von Abdera, einen Autor des späten 4. Jahrhunderts vor Christi ausschreibt. Dort heißt es:

„Die Ägypter besitzen zwei Schriften: die eine, ,demotisch genannt, lernen alle; die andere wird die, heilige' genannt. Bei den Ägyptern verstehen sie allein die Priester, die sie von den Vätern in den Mysterien lernen. ${ }^{13}$

Die Existenz zweier Schriftsysteme erklärte man sich mithilfe der Unterscheidungen zwischen dem Heiligen und dem Profanen, Priestern und Laien sowie Geheimnis und Öffentlichkeit. Spätere Quellen, insbesondere Clemens von Alexandrien und Porphyrios, erklären ganz explizit die verschiedenen Stufen des

12 Vgl. hierzu Aleida und Jan Assmann (Hrsg.): Hieroglyphen. Stationen einer anderen abendländischen Grammatologie, München 2003, und Religio Duplex, Kap. 1 (wie Anm. 4): Vom Doppelsinn der Zeichen, S. 27-62

13 Bibl. Historica III, 3, vgl. Aleida und Jan Assmann: Hieroglyphen (wie Anm. 12), S. 33. 
Schrifterwerbs, vom Demotischen zur Sakralkursive und weiter zur Hieroglyphenschrift, als Stufen eines Einweihungsweges. ${ }^{14}$

Dieser grammatologische Diskurs der Griechen bildete die wichtigste Grundlage für die seltsame Theorie der doppelten Kultur oder Religion im 17. und 18. Jahrhundert. In der Verwendung zweier verschiedener Schriften drückte sich in den Augen der Griechen eine Kluft innerhalb der ägyptischen Gesellschaft aus, die geradezu an Lessings "garstigen Graben“ denken lässt, zwischen den eingeweihten Weisen auf der einen Seite und dem Rest der schriftkundigen Gesellschaft auf der anderen. Das ist die „doppelte Philosophie“ der Ägypter, ${ }^{15}$ wie sie Heliodor und andere beschrieben: eine volkstümliche oder exoterische und eine exklusive oder esoterische, eine für das Volk, die andere für die Weisen.

Die wichtigste Quelle der Renaissance zur ägyptischen Hieroglyphenschrift, das erste Buch des Horapollon Nilotes, das aus dem 5. Jahrhundert stammt und 1419 von einem Reisenden in einem griechischen Kloster entdeckt wurde, gibt für jede einzelne von 70 Hieroglyphen nicht nur die Bedeutung an, die meistens korrekt ist, sondern auch die Motivation, die eher fantastisch anmutet. ${ }^{16}$ So soll das Schriftzeichen für "öffnen“ mit dem Bild eines Hasen geschrieben werden, weil dieser niemals die Augen schließt, und das Zeichen für „Sohn“ mit dem Bild einer Gans, weil diese einen so besonderen Familiensinn hat. Dahinter steht jene moralisierende Zoologie, die uns aus antiken Quellen wie Aelian, Plinius und dem Physiologus bekannt ist. In Wirklichkeit funktionieren die Schriftzeichen über Homophonie, aber dieses Wissen um die Lautbedeutung der Hieroglyphen ging verloren und wurde dann durch das zoologische Wissen ersetzt.

14 Clemens von Alexandria: Stromata V 4,20,3. Stromata I-VI. Hrsg. von Otto Stählin. Neu hrsg. von L. Früchtel, 4. Aufl. mit Nachträgen von U. Treu, Berlin 1985, S. 339. Siehe dazu Philippe Derchain: Les hiéroglyphes à l'époque ptolémaïque. In Claude Baurain et al. (Hrsg.): Phoinikeia Grammata, Liège 1991, S. 243-256. Porphyrios: De vita Pythagorae \$\$ $11 \mathrm{f}$. Hrsg. und übersetzt von Édouard des Places: Porphyre. Vie de Pythagore, lettre à Marcella, Paris 1982, hier S. 41, Z. 10-15.

15 Jacob Friedrich Reimmann: Idea Systematis Antiquitatis Literariae Specialioris, Hildesheim 1718. Zu Reimmann siehe Martin Mulsow und Helmut Zedelmaier (Hrsg.): Skepsis, Providenz, Polyhistorie, Tübingen 1998.

16 Horapollon: Horapollinis Nilotici Hieroglyphica Libri II. Hrsg. von Francesco Sbordone, Neapel 1940; eng. Übersetzung von George Boas: The Hieroglyphics of Horapollo, Bollingen Series XXIII, Princeton 1993; griech. und ital. Übersetzung: Horapollo: I Geroglifici. Introduzione, traduzione e note di Mario Andrea Regni e Elena Zanco, Mailand 1996; dt. Übersetzung, Buch I von Heinz-Josef Thissen: Des Niloten Horapollon Hieroglyphenbuch, Bd. 1, Leipzig, München 2001. 
Durch eine andere Entdeckung des 15. Jahrhunderts schien sich dann ein Fenster aufzutun auf den Inhalt des geheimen Wissens, das man hinter den Hieroglyphen vermutete. Das war das Corpus Hermeticum, das 1463 nach Florenz und auf das Schreibpult von Marsilio Ficino gelangte, der darüber seine Platon-Übersetzung hintanstellte: ein Konvolut von 16 theologisch-philosophischen Traktaten, die dem Weisen Hermes Trismegistos und damit dem höchsten Altertum, lange vor Platon und sogar Moses zugeschrieben wurden. ${ }^{17}$ Die Quintessenz der hermetischen Lehre lässt sich in der Formel „Hen to pan“, das All-Eine, zusammenfassen, eine Art mythologischem Pantheismus. In diesem Rahmen wird Kosmologie zu Theologie und wissenschaftliches Wissen nimmt die Eigenschaften theurgischen und magischen Wissens an, da es mit den der Natur immanenten göttlichen Kräften umgeht. Dieser praktische Zweig des Hermetismus ist als „Alchemie“ bekannt. Giordano Bruno unterstrich die magischen und mnemonischen Potentiale der Hieroglyphen im Vergleich zur Alphabetschrift:

„Von dieser Art waren die [...] Hieroglyphen oder, Heiligen Charaktere‘ bei den Ägyptern, bei denen anstelle der einzelnen bezeichnenden Zeichen [designanda] bestimmte Bilder aus den Dingen der Natur oder aus anderen Teilen genommen wurden. Solche Schriften und Sprachen kamen in Gebrauch, da durch sie die Ägypter Unterredungen mit den Göttern zum Zwecke der Ausführung wunderbarer Dinge anstrebten. Danach sind durch Teut oder einen anderen die Buchstaben erfunden worden der Art, wie wir sie noch heute in anderer Absicht verwenden. Dadurch ist der größte Schaden am Gedächtnis, an der göttlichen Wissenschaft und an der Magie entstanden." ${ }^{\text {"18 }}$

Durch diese Verbindung zwischen Hieroglyphik und Hermetismus gewann das Studium der Hieroglyphen über seine grammatologischen, semiotischen und künstlerischen Aspekte hinaus eine starke und weitreichende theologische Dimension. Das trat mit besonderer Virulenz in der Debatte um Pantheismus, Atheismus und Monotheismus hervor - alle diese Begriffe wurden im Kontext dieser Debatte zuallererst entwickelt -, die sich im Zuge des englischen Deismus und im Anschluss an die Publikation von Spinozas Ethik mit ihrer verstörenden Formel

17 Corpus Hermeticum. Hrsg. und übersetzt von Arthur Darby Nock und Andre J. Festugière, 4 Bde., Paris 1945-1954. Neuere kommentierte Übersetzungen von Brian P. Copenhaver: Hermetica. The Greek Corpus Hermeticum and the Latin Asclepius in a new English translation with notes and introduction, Cambridge 1992, und Carsten Colpe und Jens Holzhausen: Das Corpus Hermeticum Deutsch, Stuttgart, Bad Cannstatt 1997.

18 Giordano Bruno: De Magia, Op. lat., vol. III, S. 411-412. Zit. nach Elisabeth von Samsonow: Giordano Bruno, Köln 1995, S. 127 f. Vgl. Frances Yates: Giordano Bruno and the Hermetic Tradition, Chicago 1964, S. 263. 
„deus sive natura“ entfaltet hatte. Das Bild des alten Ägypten und seiner Mysterien gewann dadurch ganz neue Farben. Die ägyptische Geheimtheologie wurde jetzt als eine Art Spinozismus ante Spinozam gedeutet, und da der Spinozismus von der offiziellen Religion verteufelt wurde, erschien die Parallele zwischen dem alten Ägypten mit seinem scharfen Antagonismus zwischen Volks- und Geheimreligion und dem modernen Europa nur noch enger. ${ }^{19}$

Der topologische Aspekt der ägyptischen Initiation, dem wir uns nun zuwenden wollen, ist in der griechischen Überlieferung sehr viel weniger präsent als der grammatologische, spielt aber eine umso größere Rolle in der Vorstellung des 18. Jahrhunderts. Das wird zweifellos dem Zustrom archäologischer Anschauung verdankt in den Reiseberichten und altertumskundlichen Prachtbänden des 17. und 18. Jahrhunderts. In ihrem topologischen Aspekt nimmt die ägyptische Initiation jetzt die Züge eines Abstiegs in die Erdtiefe an. Die wichtigste antike Quelle für den topologischen Aspekt der ägyptischen Einweihung ist der verschlüsselte Bericht, den Lucius im Goldenen Esel des Apuleius von seiner Einweihung in die Isis-Mysterien gibt:

Accessi confinium mortis et calcato Proserpinae limine

per omnia vectus elementa remeavi

nocte media vidi solem

candido coruscantem lumine

deos inferos et deos superos accessi

coram

et adoravi de proxumo
Ich habe das Grenzgebiet des Todes betreten und meinen Fuß auf die Schwelle der Proserpina gesetzt

nachdem ich durch alle Elemente gefahren bin, bin ich wieder zurückgekehrt.

Mitten in der Nacht habe ich die Sonne in weißem Licht strahlen sehen. den unteren und den oberen Göttern bin ich von Angesicht zu Angesicht gegenübergetreten

und habe sie aus der Nähe angebetet. ${ }^{20}$

19 Vgl. hierzu mein Buch: Moses der Ägypter. Entzifferung einer Gedächtnisspur, München 1998. Engl. Übersetzung: Moses the Egyptian. The Memory of Egypt in Western Monotheism, Cambridge, Mass. 1997.

20 Vgl. hierzu besonders Reinhold Merkelbach: Isis-Regina, Zeus-Sarapis, Stuttgart, Leipzig 1995, Kap. 23, S. 266-303, John Gw. Griffiths: Apuleius of Madauros. The Isis-Book. Me- 
Drei Motive dieser Passage kehren in den Texten des 18. Jahrhunderts immer wieder: die Verbindung von Initiation und Tod, die Reise durch die vier Elemente und die Schau der Götter.

Der erste neuere Autor, der diese knappen Anspielungen in eine detaillierte Erzählung der ägyptischen Einweihung umgesetzt hat, war der Abbé Jean Terrasson, Gräzist am Collège de France und renommierter Herausgeber der Historischen Bibliothek des Diodor von Sizilien. In seinem 1731 erschienen Roman Séthos, ${ }^{21}$ widmete er gute 100 Seiten der Beschreibung der Einweihung seines Helden in die Mysterien der Isis. Séthos ist der legitime Thronerbe, der aber von seiner bösen Stiefmutter Daluca zugunsten ihrer eigenen Kinder unterdrückt wird. Als er 16 Jahre alt wurde, gibt sein Mentor Amédès endlich seinem glühenden Wunsch nach, in die Mysterien eingeweiht zu werden. Séthos und Amédès betreten die Große Pyramide des Nachts auf der Nordseite und steigen einen sehr tiefen Schacht hinab. Unten gelangen sie nach weiteren Schächten, Hallen und Korridoren endlich an ein Portal mit einer Inschrift, die wörtlich im Libretto der Zauberflöte wiederkehrt. In der von Mozart und Schikaneder benutzten Übersetzung von Matthias Claudius lautet sie:

„Wer diesen Weg allein geht und ohne hinter sich zu sehen, der wird gereinigt werden durch das Feuer, durch das Wasser und durch die Luft; und wenn er das (!) Schrecken des Todes überwinden kann, wird er aus dem Schooß der Erde wieder herausgehen, und das Licht wieder sehen, und er wird das Recht haben, seine Seele zu der Offenbarung der Geheimnisse der großen Göttin Isis gefasst zu machen! ${ }^{{ }^{22}}$

Amédès muss zurückbleiben und Séthos muss seinen Weg allein fortsetzen. Er wird - nicht von zwei, sondern - von drei Geharnischten empfangen, die ihn warnen, dass es von hier ab kein Zurück gibt. Nach weiteren Stationen muss er schließlich einen Raum voll lodernden Feuers durchqueren und einen Kanal mit kaltem Wasser durchschwimmen. Die letzte Prüfung bzw. das letzte Element, dass

tamorphoses, Book XI, Leiden 1975, S. 296-308, Jan Bergman: Per omnia vectus elementa remeavi. In: Ugo Bianchi und Maarten Jozef Vermaseren (Hrsg.): La soterologia degli culti orientali nell' impero romano, Leiden 1982, S. 671-702, Erik Hornung: Altägyptische Wurzeln der Isismysterien. In: Hommages à Jean Leclant IV, Kairo 1994, S. 287-293; Françoise Dunand: Isis, mère des dieux, Paris 2000, S. 127-140.

21 Séthos. Histoire ou vie, tirée des monuments, Anecdotes de l'ancienne Égypte; Ouvrage dans lequel on trouve la description des Initiations aux Mystères Égyptiens. Traduit d'un manuscrit Grec, Paris 1731.

22 Matthias Claudius: Geschichte des egyptischen Königs Sethos, Breslau 1777-78, S. 155. 
er durchqueren muss nach Erde - der gefährliche Abstieg - Feuer und Wasser ist die Luft: er muss zwei Ringe ergreifen, die ihn unter enormem Getöse durch die Luft direkt in den Tempel des Ptah und vor eine Gruppenstatue von Isis, Osiris und Horus befördern. Dort wird er vom Hohepriester mit einem Gebet begrüßt, das ebenfalls in die Zauberflöte übernommen wurde:

„O Isis, große Göttin der Egypter, gieb deinen Geist dem neuen Diener, der so viel Gefahren und Beschwerlichkeit überstanden hat, um vor dir zu erscheinen. Mache ihn auch sieghaft in den Proben seiner Seele, und lehre sein Herz deine Gesetze, damit er würdig werde, zu deinen Geheimnissen zugelassen zu werden. Als alle Priester die ersten Worte dieses Gebets: O Isis, große Göttin der Egypter, wiederholt hatten, ließ man dem Sethos wieder aufstehn, und der Oberpriester reichte ihm ein zusammengesetztes Getränk, das die Griechen Cyceon genannt haben, und sagte ihm, daß dies ein Getränk der Mnemosyne oder des Gedächtnisses für alle die Lehren sey, die er von der Weisheit erhalten werde. ${ }^{\text {23 }}$

Terrasson präsentierte seinen Roman als Übersetzung eines griechischen Manuskripts, das er überdies mit gelehrten Fußnoten ausstattete. Wegen seines großen Rufs als Gelehrter wurde sein Bericht der ägyptischen Einweihung fast allgemein als authentisch aufgefasst. So kam es zu der Vorstellung eines von unterirdischen Anlagen, die der geheimen Religion dienten, vollständig unterminierten Ägypten und der Einweihung als eines Übergangs aus der oberirdischen Welt der Illusionen in die unterirdische Welt der Wahrheit, wobei auch noch die Idee der hermetischen Höhle eine große Rolle spielte, in der Hermes Trismegistos die von ihm mit der über die Sintflut geretteten Urweisheit beschrifteten Säulen verborgen habe. ${ }^{24}$

\section{III}

Das alte Ägypten erschien jetzt in der Optik einiger Geheimgesellschaften nicht nur als der Ursprung aller antiken Staaten und Religionen einschließlich des biblischen Monotheismus, sondern auch als der Spiegel der modernen Gesellschaft mit ihrer Außenseite von Kirche und Absolutismus und ihrer Innenseite von Deismus und aufgeklärter Philosophie. Die Freimaurer betrachteten sich als Erben der

23 Ebd., S. 165.

24 Vgl. hierzu Jan Assmann: Die Zauberföte. Oper und Mysterium, München 2005, S. 106112. 
ägyptischen Priester und der antiken Mysterienreligionen, und die ägyptischen Mysterien wurden jetzt erforscht als Modelle der modernen Freimaurerei. Das aktivste Zentrum dieser Mysterienforschung war die den Illuminaten besonders verbundene Wiener Loge zur Wahren Eintracht, die die systematische Erforschung der Mysterien zu ihrem zentralen Projekt machte und in den Jahren 1784 und 1787 nicht weniger als 14 Artikel und längere Abhandlungen zu diesem Thema veröffentlichte. ${ }^{25}$ Einer der für unsere Frage nach dem topologischen Aspekt der ägyptischen Einweihung interessantesten Beiträge ist ein anonymer Essay über die Frage der wissenschaftlichen Freimaurerei. ${ }^{26}$ Der Beitrag wird allgemein Ignaz von Born zugeschrieben, dem Großmeister der Loge, aber nach Aussage des Sitzungsprotokolls ${ }^{27}$ hat ein gewisser Anton Kreil über genau diesen Gegenstand in den Sitzungen vom 16. und 22. April 1785 zwei Vorträge gehalten, und es kann nicht der geringste Zweifel daran bestehen, dass er der Verfasser des besagten Artikels ist. Kreil war ein hochgebildeter Philosoph und Altphilologe, der verschiedene besonders interessante Artikel zu dem Journal beisteuerte und auch der Schöpfer der Unterscheidung zwischen "wissenschaftlicher" und "religiöser Freimaurerei“. Wissenschaftliche Freimaurerei wurde als eine Bastion zur Beförderung der Aufklärung definiert und religiöse Freimaurerei als eine Form von spirituellem Konservativismus und Geheimniskrämerei. ${ }^{28}$ Der interessanteste Aspekt dieser Vorträge ist aber das Datum, denn laut Protokoll wurden sie in den beiden Sitzungen gehalten, in denen Leopold Mozart zum Gesellen und Meister erhoben wurde, in Anwesenheit von Wolfgang. Damit ist der definitive Beweis erbracht, dass Mozart von den Mysterienforschungen der Loge Kenntnis hatte. Kreils Vorträge erweisen sich als die farbigste und eindrucksvollste Darstellung der ägyptischen Mysterien in ihrem topologischen Aspekt, denn er gehört zu den ersten, die den archäologischen Quellen, das heißt Reiseberichten und Bildbänden Rechnung tragen. Indem er nun diese Berichte mit der Theorie der ägyptischen Doppelkultur in Verbindung bringt, kommt er zu dem Schluss, dass diese ausgedehnten unterirdischen Anlagen, von denen ganz Ägypten unterminiert sein soll, Gänge, Korridore, Pfeilerhallen, Lichthöfe, Treppen und Schächte, alles von oben bis unten mit

25 Vgl. Religio Duplex (wie Anm. 4), S. 242-348.

26 In: JF 7, 1985, S. 49-78.

27 Heinz-Josef Irmen: Die Protokolle der Wiener Freimaurerloge „Zur Wahren Eintracht, 1781-1785, Frankfurt a. M. 1994, S. 271, Nr. 374, und S. 272, Nr. 376. Irmen schreibt die Rede Ignaz von Born zu „anläßlich einer Meistererhebung“. Vgl. S. 26.

28 Jan Snoek: What Means the Word „Religious“ in Reinhold's Religious Freemasonry. In Sibylle Appel (Hrsg.): Egypt - Temple of the Whole World. Ägypten - Tempel der ganzen Welt, Leiden 2003, S. 409-420. 
Hieroglyphen bedeckt, nur einem Zweck dienen konnten, nämlich als Bibliothek, Laboratorium und Ritualbühne zur Pflege und Weitergabe der geheimen Religion und ihrer geheimen Theologie der Natur. Die ägyptischen Priester verbrachten ihre meiste Lebenszeit unter der Erde mit dem Studium der Geheimnisse von Natur und Theologie und hüllten ihr Wissen in den undurchdringlichen Schleier der Hieroglyphen und der verborgenen Räume. Sie waren darin so erfolgreich, meint Kreil, dass ihre Tradition an manchen Orten bis heute lebendig ist. Man kann sich nun gut vorstellen, dass Mozart, der ja ein sehr engagierter Freimaurer war, ${ }^{29}$ bei dieser Gelegenheit die Idee kam, die ägyptischen Mysterien auf die Bühne zu bringen und das Prinzip der doppelten Religion in die Form einer Doppeloper zu übersetzen mit einer volkstümlichen Außen- und einer philosophisch-geheimnisvollen Innenseite. Als dann sechs Jahre später Emanuel Schikaneder sich von ihm eine Oper wünschte, fügten die beiden das Zauberflötenmärchen aus Wielands Dschinnistan und Mozarts Vorstellung der ägyptischen Einweihung zu einer opera duplex aus Volkstheater und Mysterienspiel zusammen und schufen damit die überzeugendste ästhetische Darstellung der ägyptischen Initiation. ${ }^{30}$

Im Licht der Mysterientheorie mit ihrem Antagonismus von außen und innen, oben und unten, Volksreligion und Geheimreligion, Illusion und Wahrheit, lösen sich alle Schwierigkeiten der Zauberfötenhandlung, die früh schon zu der Vorstellung führten, Mozart und Schikaneder hätten mitten in der Arbeit ihren Plan geändert, aus der guten Königin der Nacht eine Rachefurie und aus dem Bösewicht Sarastro einen weisen Herrscher gemacht und den Plot von der Rettung der geraubten Prinzessin in ein Einweihungsritual umgelenkt. Wenn man die

29 Mozarts Beziehungen zur Freimaurerei reichen über befreundete Salzburger Familien weit in seine Jugendzeit zurück und müssen sich in den Jahren 1773-79 erheblich intensiviert haben, als er die Bühnenmusik für das Theaterstück des prominenten Freimaurers Philipp Freiherrn von Gebler „Thamos. König in Ägypten“ schrieb bzw. überarbeitete. Selbst einer Loge angeschlossen hat er sich jedoch erst in seiner Wiener Zeit. Am 14.12. des Jahres 1784 trat er der von seinem Freund aus Mannheimer Tagen, dem Bühnenschriftsteller, Dramaturgen und Theatertheoretiker Otto Reichsfreiherrn von Gemmingen-Hornberg, gegründeten Loge „Zur Wohltätigkeit “ bei und wurde bereits am 5.1.1785 in der Schwesterloge „Zur Wahren Eintracht “ zum Gesellen und wenig später zum Meister befördert. Über Mozart als Freimaurer und die Wiener Logen zwischen 1780 und 1791 vgl. Howard Chandler Robbins Landon: Mozart and the Masons, London 1982, Jan Assmann: Die Zauberföte. Oper und Mysterium, München 2005, S. 149-154, ders.: Mozart und die Wiener Freimaurerei. In Claudia Maria Knispel und Gernot Gruber (Hrsg.): Mozarts Welt und Nachwelt. Das Handbuch, Laaber 2009, S. 148-160.

30 Zur Entstehungsgeschichte der Zauberföte vgl. Jan Assmann: Schikaneder, Mozart und die Zauberflöte. Nachwort zu Jan Assmann (Hrsg.): Die Zauberflöte. Ein literarischer Opernbegleiter, Köln 2012. 
Geschichte als eine Mysterienreise versteht, die den Initianden - Tamino - von der Oberwelt der Illusionen (dem Reich der Königin der Nacht) durch verschiedene Prüfungsstationen der Unterwelt in die Sphäre der Eingeweihten bzw. Aufgeklärten führt, wird der Sinn der Wende klar, die die Geschichte nimmt. Nach der Theorie Warburtons und der Wiener Freimaurer führt der Einweihungsweg von den Illusionen der Volksreligion durch das Dunkel der Desillusionierung und Prüfung schließlich zum vollen Licht der Wahrheit und verläuft in vier Stadien: das Ausgangsstadium des Irrtums, die Reinigung von falschen Vorstellungen, die kleinen Mysterien und die großen Mysterien. Zu den kleinen Mysterien, die aus Prüfungen und Belehrungen bestehen, ist jeder unbescholtene, freie Bürger zugelassen. Nur sehr wenige dagegen, die zu Herrschern berufen sind oder sonst durch Tugend und Weisheit hervorragen, sind zu den Großen Mysterien zugelassen.

Die Großen Mysterien konfrontieren den Initianden mit dem Tod und versetzen ihn in echte Todesangst. Plutarch beschreibt in einem von Warburton und den Freimaurern viel zitierten Fragment die Erfahrung der Einweihung in die Großen Mysterien als eine echte Nah-Tod-Erfahrung:

„Hier ist die Seele ohne Erkenntnis außer wenn sie dem Tode nah ist. Dann aber macht sie eine Erfahrung, wie sie jene durchmachen, die sich der Einweihung in die Großen Mysterien unterziehen. Daher sind auch das Wort ,sterben' ebenso wie der Vorgang, den es ausdrückt, $(\tau \varepsilon \lambda \varepsilon v \tau \alpha \nu)$ und das Wort ,eingeweiht werden ${ }^{\circ}\left(\tau \varepsilon \lambda \varepsilon ı \sigma \alpha_{\iota}\right)$ ebenso wie damit bezeichnete Handlung einander gleich. Die erste Stufe ist nur mühevolles Umherirren, Verwirrung, angstvolles Laufen durch die Finsternis ohne Ziel. Dann, vor dem Ende, ist man von jeder Art von Schrecken erfaßt, und alles ist Schaudern, Zittern, Schweiß und Angst. Zuletzt aber grüßt ein wunderbares göttliches Licht und man wird in reine Gefilde und blühende Wiesen aufgenommen, wo Stimmen erklingen und man Tänze erblickt, wo man feierlich-heilige Gesänge hört und göttliche Erscheinungen erblickt. Unter solchen Klängen und Erscheinungen wird man dann, endlich vollkommen und vollständig eingeweiht, frei und wandelt ohne Fesseln mit Blumen bekränzt, um die heiligen Riten zu feiern im Kreise der heiliger und reiner Menschen. ${ }^{\text {"31 }}$

Wenn wir dieses Schema auf die Oper anwenden, stellen wir eine überraschende Übereinstimmung fest. Die Einteilung in kleinere und grosse Mysterien entspricht

31 Plutarch fr. 178 Sandbach. Vgl. dazu u. a. Paolo Scarpi (Hrsg.): Le religioni dei Misteri, Milano 2002, Bd. 1, S. 176 f. mit italienischer Übersetzung, außerdem Walter Burkert: Antike Mysterien, München 1990, S. 77. 
genau dem ersten und zweiten Teil des Zweiten Akts. Die Oper gliedert sich in zwei Aufzüge, die ihrerseits noch einmal in zwei Abschnitte ungefähr gleicher Länge unterteilt sind: eine Abfolge von Gesangsnummern und gesprochenen Dialogen (erster und dritter Abschnitt) wird jeweils gefolgt von einem Finale, in dem nur gesungen und nicht gesprochen wird (zweiter und vierter Abschnitt). Jeder Abschnitt endet überdies in der Tonart, in der er beginnt: Es-Dur (Ouvertüre bis Duett „Bei Männern...“), C-Dur („Zum Ziele führt dich“ bis Chor „Wenn Tugend und Gerechtigkeit“), F-Dur (Priestermarsch bis Arie „Ein Mädchen oder Weibchen“) und wieder Es-Dur („Bald prangt“ bis Schlusschor). Der dritte Teil, also der erste Teil des Zweiten Akts, enthält die typischen Prüfungen und Instruktionen der kleinen Mysterien. Hier ist auch Papageno dabei, wenn er auch eine eher klägliche Figur abgibt. Es geht hier um Selbstkontrolle, die Initianden müssen lernen, ihrem Kommunikationstrieb zu widerstehen, und während Papageno es nicht schafft, gegenüber den drei Damen und der als Altes Weib verkleideten Papagena zu schweigen, behält Tamino seine Fassung gegenüber der geliebten Pamina trotz ihrer Verzweiflung und Todesabsicht. Der vierte Teil gilt den Großen Mysterien. Hier ist Papageno nicht dabei, aber Pamina vereint sich mit Tamino zur Feuer- und Wasserprobe. Von Tod ist ständig die Rede in diesem Teil, der mit Paminas versuchtem Selbstmord beginnt und dann Tamino vor den "Schrekkenspforten“ zeigt, „die Not und Tod ihm dräun“. „Wenn er des Todes Schrecken überwinden kann“, heißt es in der Inschrift, „schwingt er sich von der Erde himmelan.“ „Ein Weib, das Nacht und Tod nicht scheut", singen die Geharnischten und Tamino, „ist würdig und wird eingeweiht.“ „Wir wandeln durch des Tones Macht", singen die beiden „froh durch des Todes düstre Nacht“. In den Großen Mysterien geht es um die Verwandlung des Initianden durch die Erfahrung der Todesnähe, das heißt des „Erhabenen“.32

Wenn man die zwei Teile des Zweiten Akts den kleinen und großen Mysterien zugeordnet hat, erkennt man leicht die Stadien der Illusion und der Desillusionierung in den beiden Teilen des Ersten Akts. Tamino - und mit ihm die Zuschauer - werden zunächst ganz eingenommen von der Erzählung der Königin der Nacht mit ihrer Geschichte von der entführten Prinzessin und müssen dann im zweiten Teil eine gründliche Umorientierung durchmachen, die Tamino von den falschen Vorannahmen befreit, aber ihn im Übrigen in völligem Dunkel lässt. „O ew'ge Nacht, wann wirst du schwinden?" Offensichtlich will die Oper die verschiedenen

32 Vgl. hierzu meinen Aufsatz: Über das Erhabene. Schiller im Licht von Kant und Mozart. Marbacher Schillerrede 2006. In: Schiller Jahrbuch 51, 2007, S. 166-182, und Christine Pries (Hrsg.): Das Erhabene. Zwischen Grenzerfahrung und Größenwahn, Berlin 1989. 
Stadien der ägyptischen Einweihung in ihren Gegensätzen auf die Bühne bringen, von Volksreligion und esoterischer Weisheit, Aberglauben und Aufklärung, der Oberwelt der Illusionen und der Unterwelt der wahren Erkenntnis.

Nach den frühen Bühnenbildern ${ }^{33}$ zu urteilen, spielt die Zauberflöte auch gar nicht im alten Ägypten, sondern in einer exotischen Gegend oder einem Freimaurer-Park, wo die Isismysterien noch begangen werden. Nicht Altägypten, sondern die ägyptischen Mysterien - von denen Kreil ja behauptete, dass sie irgendwo noch lebendig wären - bilden das eigentliche Thema der Oper. Ins alte Ägypten wurde die Oper von den Bühnenbildnern erst ab 1815 verlegt, als die ersten Bände der Description de l'Egypte erschienen waren. ${ }^{34}$

\section{IV}

Überraschenderweise gibt es nun auch eine enge Verbindungslinie, die von der Mysterienforschung der Wiener Loge zu Schillers Ballade Das verschleierte Bild zu Sais führt. Ein sehr aktives Mitglied dieser Loge, der junge Philosoph Carl Leonhard Reinhold, hatte sich im November 1784, kurz vor Mozarts Eintritt, nach Leipzig abgesetzt, um dort zu promovieren, wurde aber noch vorher auf eine Professur nach Jena berufen. Dort arbeitete er jedoch weiter an dem Mysterienprojekt und steuerte zum Journal zwei lange Abhandlungen bei, die er dann unter dem Titel Die hebräischen Mysterien oder Die älteste religiöse Freimaurerei als Buch veröffentlichte. ${ }^{35}$ Schiller, den Reinhold als Kollegen nach Jena holte, war von diesem Buch so beeindruckt, dass er einen Essay mit dem Titel Die Sendung Moses veröffentlichte, der praktisch eine Kurzfassung des Reinholdschen Buches darstellt. ${ }^{36}$ Reinhold war eine führende Figur, nicht nur des deutschen Idealismus, sondern auch des Illuminatenordens. Sein Buch basiert auf einer gründlichen Lektüre vor allem von Warburton und Spencer und vertritt eine erstaunliche Neuauflage von

33 Vgl. Annette Frese: „Das Theater verwandelt sich.... Bühnenbilder, Figurinen und Illustrationen zur Zauberflöte. In Bärbel Pelker (Hrsg.): Theater um Mozart, Heidelberg 2006, S. $143-205$.

34 Die „Description de l'Égypte“ erschien unter der Leitung von François Jomard zwischen 1809 und 1828 und umfasste neun Text- und 11 Bildbände.

35 Carl Leonhard Reinhold: Die hebräischen Mysterien oder die älteste religiöse Freymaurerey (1787). Hrsg. und eingeleitet von Jan Assmann, Neckargemünd, 2. Aufl., 2006, zuerst 2001.

36 Friedrich Schiller: Die Sendung Moses. In Friedrich Schiller, Werke und Briefe. Hrsg. von Otto Dann, Bd. VI: Historische Schriften und Erzählungen, Frankfurt a. M., 2000, S. 451474. 
Spencers These vom ägyptischen Ursprung des mosaischen Monotheismus. Moses, das ist auch Reinholds Ansicht, lernte alles über die wahre Religion in den ägyptischen Mysterien, in die er als Prinz eingeweiht worden war; die Religion, die er dann den Hebräern verkündete, ist nichts als eine popularisierte Version des ägyptischen esoterischen Deismus. Die ägyptische Gottheit ist das All-Eine, die verschleierte Gottheit von Sais, deren Inschrift verkündet: „Ich bin alles, was da war, ist, und sein wird; kein Sterblicher hat meinen Schleier gelüftet. ${ }^{\text {"37 Diese }}$ Gottheit hat keinen Namen, wie es im Traktat Asclepius des Corpus Hermeticum heißt, da sie einzig ist und zugleich alles, so dass sie entweder mit allen oder gar keinem Namen benannt werden kann. ${ }^{38}$ Diese Namenlosigkeit, ergänzt Schiller, ist der Inbegriff des Erhabenen. Moses übersetzt dieses Konzept in "Jehova“, was ebenfalls kein Name, sondern der Entzug eines Namens ist: „Ich bin der ich bin“. Schiller und Reinhold folgen der Septuaginta-Fassung - ego eimi ho ön - was sie als „Ich bin das wesentliche Sein“ übersetzen und im Sinne von „Ich bin was da ist" verstehen.

Beethoven, der Schillers Essay im Jahre 1825 las, hat sich die Kernsätze der vermeintlich ägyptischen Arcantheologie auf einem Blatt abgeschrieben, das er sich hinter Glas gerahmt auf seinen Schreibtisch stellte. ${ }^{39}$

37 "ego eimi pan to gegonos kai on kai esomenon" - Plutarch: De Iside et Osiride, Kap. 9 (354C). Hrsg von. John Gw. Griffiths, Cardiff 1970, S. 130 f. und 283 f.

38 Ps. Apul. Ascl. cap. 20 = Corpus Hermeticum. Hrsg. von Arthur Darby Nock und Andre J. Festugière II, 320f. „Der Schöpfer ist namenlos oder trägt jeden Namen, weil er ja einer und alles ist, so daß man entweder alles mit seinem Namen oder ihn selbst mit den Namen von allem benennen muß."

39 Siehe hierzu Erhart Graefe: Beethoven und die ägyptische Weisheit. In: Göttinger Miszellen 2, 1972, S. 19-21 mit Verweis auf Anton F. Schindler: Biographie von Ludwig van Beethoven, 3. Aufl., Münster 1860, S. 161. In der von Ignaz Moscheles herausgegebenen und übersetzten englischen Ausgabe von 1841 „The Life of Beethoven“, Mattapan 1966, Bd. 2, S. 163, heißt es hierzu und hinsichtlich von Beethovens religiösen Überzeugungen: „If my observation entitles me to form an opinion on the subject, I should say he (scil. Beethoven) inclined to Deism; in so far as that term might be understood to imply natural religion. He had written with his own hand two inscriptions, said to be taken from a temple of Isis “. Beethovens Text, den Schindler in Faksimile wiedergibt, lautet: „Ich bin, was da ist / Ich bin alles, was ist, was war, und was seyn wird, kein sterblicher Mensch hat meinen Schleyer aufgehoben / Er ist einzig von ihm selbst, u. diesem Einzigen sind alle Dinge ihr Daseyn schuldig “. Die Sätze sind durch doppelte Schrägstriche voneinander abgesetzt. Der dritte ist vielleicht später zugefügt; die Handschrift wirkt kleiner und flüchtiger. Beethoven war kein Freimaurer, hatte aber enge Freunde unter Freimauerern und Illuminaten, zu denen etwa Beethovens Lehrer Christian Gottlob Neefe gehörte. 
Aber weil nun einmal ein Staat und eine zivile Gesellschaft nicht auf natürliche Theologie gegründet werden können, musste Moses seine erhabene Idee der Fassungskraft des Volkes anpassen und den Gott der ägyptischen Philosophen in einen Nationalgott, den biblischen Gott der Väter, und natürliche in politische Theologie übersetzen. Auch hier aber erhielt sich unter den Weisen eine Spur der Wahrheit, so dass auch die jüdische Religion eine Doppelstruktur, und zwar die Form der religiösen Freimaurerei annahm. Schiller spricht in diesem Zusammenhang von einem Betrug in edler Absicht. Man fühlt sich an Schönbergs Oper Moses und Aron erinnert, in der dieser Konflikt zwischen philosophischer Wahrheit und fasslicher Religion auf zwei Figuren verteilt wird, Moses und Aron. ${ }^{40}$

Wie Moses in Schönbergs Oper an der Unkommunizierbarkeit der Wahrheit, so scheitert der Jüngling in Schillers Ballade Das verschleierte Bild zu Sais an ihrer Unfassbarkeit. ${ }^{41}$ Der Fehler des Jünglings, der von „des Wissens heißem Durst“ getrieben nach Sais eilt, um sich in die Mysterien der Isis einweihen zu lassen, liegt darin, dass er die vorbereitenden Stufen der Einweihung, die Desillusionierung und die kleinen Mysterien überspringt. Er will sich sofort der letzten Schau aussetzen, ohne zu ahnen, dass nur der Vorbereitete, Auserwählte, den Anblick der Wahrheit ertragen kann; für den Unvorbereiteten bedeutet er durch die plötzliche Zerstörung aller lebensdienlichen Illusionen die Konfrontation mit dem Nichts, dem Abgrund. ${ }^{42}$ Das ist die Struktur des Erhabenen. Es ereignet sich nur in der Konfrontation zwischen dem Furchtbaren und der "standhaltenden“ Seele. ${ }^{43}$ Es ereignet sich nicht im schieren Grauen, dem nichts standhält, und ebenso wenig in der von starken Gewissheiten, etwa der Unsterblichkeit, getragenen Seele, die das Grauen gar nicht verspürt. Was der noch nicht desillusionierte bzw. aufgeklärte, noch nicht in jahrelangem Lernen und Leiden vorbereitete und erprobte Jüngling erblickt, ist eine Wahrheit, der sein Geist nicht standzuhalten vermag, weil er sie nur als Nichts, als Abgrund der Negation, als schiere und unvorstellbare Sinnlosigkeit erlebt. Schiller und Kant definierten das Erhabene als eine überwältigende Erfahrung, die entweder den physischen Überlebenswillen oder die geistige Fas-

40 Vgl. hierzu meinen Aufsatz: Die Mosaische Unterscheidung in Schönbergs „Moses und Aron“. In: Musik und Aesthetik 9, Heft 33, 2005, S. 5-29.

41 Vgl. hierzu meine Studie: Das verschleierte Bild zu Sais. Schillers Ballade und ihre griechischen und ägyptischen Hintergründe. In: Lectio Teubneriana VIII, Stuttgart und Leipzig 1999.

42 In der genannten Studie habe ich die von dem vorwitzigen Jüngling geschaute Wahrheit mit dem Tod identifiziert, unter Berufung auf den Satz Ludwig Börnes: „Hinter dem Schleyer der Isis lauscht der Tod." (Über Freimaurerei).

43 Christine Pries (Hrsg.): Das Erhabene (wie Anm. 32). 
sungskraft in höchstem Maße herausfordert. Beide erblickten im verschleierten Bild zu Sais und seiner Inschrift den Inbegriff des Erhabenen.

Für die Illuminaten und andere der Aufklärung verschriebene Geheimgesellschaften muss es jedenfalls als eine bedeutende Bestätigung erschienen sein, dass sich ihre religiösen Überzeugungen, diese Verbindung von Deismus, Spinozismus, Pantheismus, auf die ägyptischen Mysterien und die Urreligion der Menschheit zurückführen lassen. Ignaz von Born, prominenter Mineraloge, Illuminat und Meister vom Stuhl der Wiener Loge zur Wahren Eintracht, schrieb in seiner Abhandlung Über die Mysterien der Aegyptier, mit der er die Serie der Mysterienschriften im Journal für Freymäurer eröffnete:

„Die Kenntniß der Natur ist der Endzweck unsrer Anwendung. Diese Zeugerinn, Nährerinn und Erhalterinn aller Geschöpfe verehren wir unter dem Bilde der Isis. - Nur jener deckt ihren Schleyer ungestraft auf, der ihre ganze Macht und Kraft kennet. ${ }^{{ }^{4} 4}$

Auch Kant verbindet in jener Fußnote seiner dritten Kritik, in der er das verschleierte Bild zu Sais als den Gipfel des Erhabenen preist, das Bild der Isis mit der Naturwissenschaft:

„Vielleicht ist nie etwas Erhabeneres gesagt oder ein Gedanke erhabener ausgedrückt worden als in jener Aufschrift über dem Tempel der Isis (der Mutter Natur): ,Ich bin alles was da ist, was da war und was da sein wird, und meinen Schleier hat kein Sterblicher aufgedeckt.' Segner benutzte diese Idee, durch eine sinnreiche, seiner Naturlehre vorgesetzte Vignette, um seinen Lehrling, den er in diesen Tempel einzuführen bereit war, vorher mit dem heiligen Schauer zu erfüllen, der das Gemüth zu feierlicher Aufmerksamkeit stimmen soll. ${ }^{45}$

Damit verweist Kant zum einen auf die von Schiller betonte emotionale Dimension des Erhabenen - ohne Schrecken und Schaudern ist der Tempel der Natur nicht zu betreten - und zum anderen auf die ikonographische Tradition, die sich mit Isis als „Mutter Natur“ verbindet. Segners Vignette (Abbildung 1) stellt in der Tat die Göttin Isis dar, kenntlich an dem Sistrum, das sie in der Hand hält, und sie ist verschleiert. In ihrem Rücken vermessen zwei Putten ihre Fußspuren, denn sie können sie nicht direkt, von vorn, studieren. Das ist eine Anspielung auf die

44 Ignaz von Born: Über die Mysterien der Aegyptier. In: Journal für Freymäurer 1, 1784, S. 15-132. Er zitiert als seine Quelle Plutarch.

45 Immanuel Kant: Kritik der ästhetischen Urteilskraft. In: Werke in 10 Bänden. Hrsg. von Wilhelm Weischedel, Bd. VIII, Darmstadt 1968, S. 417. 


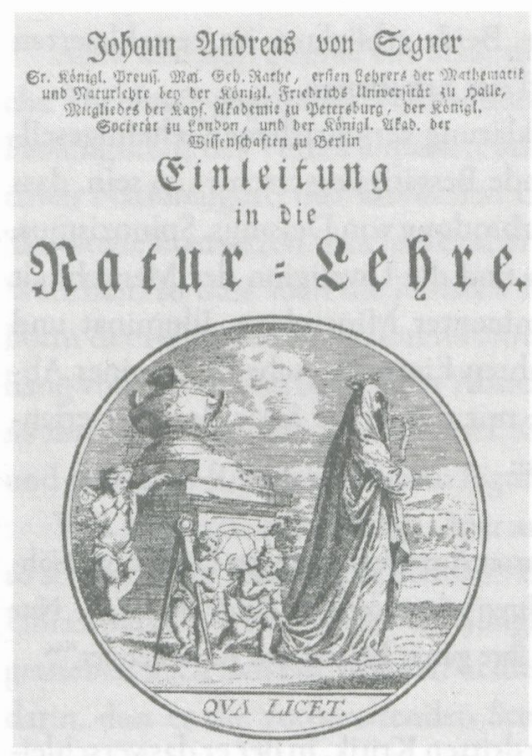

Abb. 1: Titelblatt zu J. A. v. Segner, Einleitung in die Naturlehre, Göttingen 3. Aufl. 1770

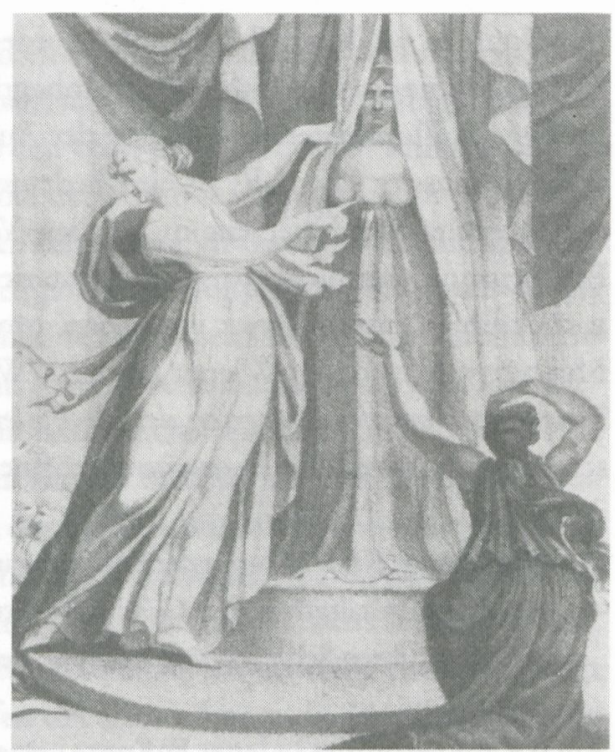

Abb. 2: Heinrich Füßli, Frontispiz zu Erasmus Darwin, The Temple of Nature, Edinburgh 1809

Szene in Exodus 33: 20-23. Moses wünscht sich, Gottes Antlitz zu sehen. Der aber verwehrt es ihm mit den Worten „kein Mensch kann mein Antlitz sehen und leben“. Der Anblick Gottes sei nur von hinten möglich. Dafür kommt aber das Motiv der verschleierten und entschleierten Isis durchaus häufig auf anderen naturkundlichen Werken des 17. und 18. Jahrhunderts vor. ${ }^{46}$

Das bei weitem eindrücklichste Bild dieser Art, das Heinrich Füssli dem naturkundlichen Lehrgedicht The Temple of Nature von Erasmus Darwin, dem Großvater von Charles, voranstellt, bringt wirklich den Schrecken des Erhabenen und den heiligen Schauer zum Ausdruck, von dem Kant und Schiller schreiben (Abbildung 2). Darauf sieht man eine Frau, von hinten, die mit Gesten des Schreckens und vielleicht auch Entzückens auf den Anblick der vielbrüstigen Isis reagiert, von der eine Priesterin abgewandten Gesichts den Vorhang oder Schleier wegzieht. Hier geht es nun wirklich um einen Tempel, The Temple of Nature, und um eine Einweihung. Was Füssli darstellt, ist der letzte und höchste Schritt der Initiation, die Epoptie oder Schau, bei dem einzelnen Erwählten dann doch der allen ge-

46 Pierre Hadot: Le voile d'Isis, Paris 2004. 
Abb. 3: Gedenkmedaille zum Erscheinen

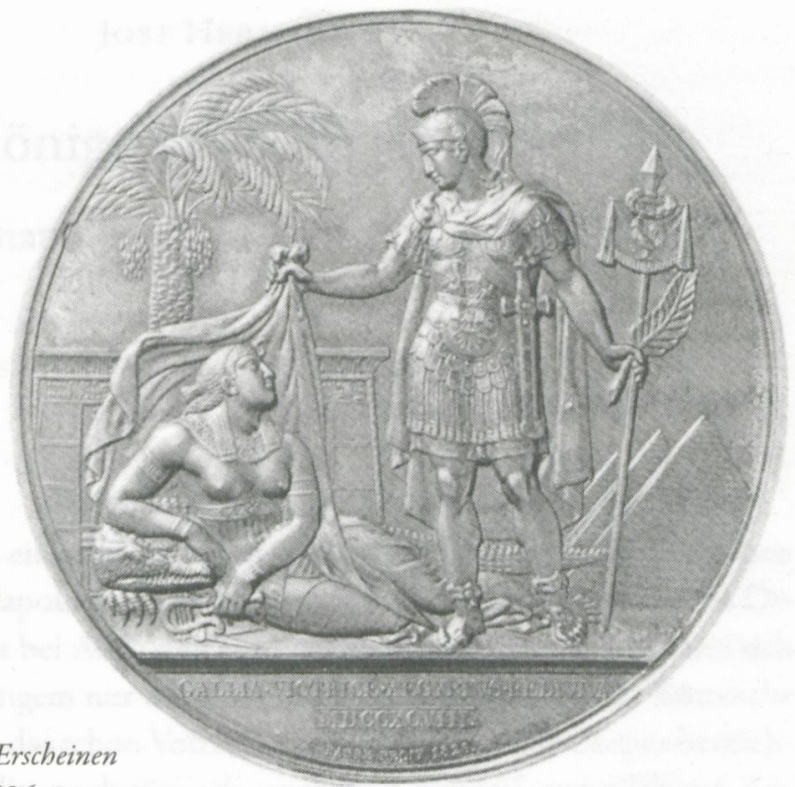

der Description de l'Egypte, 1826

wöhnlichen Sterblichen entzogene Anblick der Gottheit gewährt wird. Diese Szene ist der Inbegriff, gewissermaßen die „Urszene“ des Erhabenen.

Das Motiv der Entschleierung der Isis bringt auf besonders augenfällige Weise das sogenannte Projekt der Moderne zum Ausdruck, und zwar in dem zentralen Aspekt einer Dekonstruktion des Geheimnisses. Das betrifft vor allem auch die Ägyptologie, die mit den Ideen der ägyptischen Mysterien, der Hieroglyphen als symbolischer Geheimschrift und der Gräber als Stätten der Geheimreligion aufgeräumt hat. Eine Gedenkmedaille zum Abschluss des Monumentalwerks der Description de l'Egypte stellt einen römischen Soldaten als Symbol des siegreichen napoleonischen Frankreich (Gallia victrix) dar, der den Schleier von der „wieder belebten“" (rediviva) Personifikation des Alten Ägypten hebt (Abbildung 3). 